$>$ Depuis la dérivation des premières lignées de cellules souches embryonnaires pluripotentes chez la souris au début des années 1980, une pléthore de lignées a été obtenue chez diverses espèces de mammifères, dont les rongeurs, les lagomorphes ${ }^{1}$ et les primates. Ces lignées se distinguent par leurs caractéristiques moléculaires et fonctionnelles et correspondent aux différents états de pluripotence observés chez l'embryon, entre les stades blastocyste et gastrula. Ces lignées se répartissent le long d'un gradient, ou continuum de pluripotence, dont les deux extrémités sont symbolisées par les états appelés naïf et amorcé. Les cellules souches pluripotentes humaines sont dans un état de pluripotence amorcé (au bas du gradient), une position qui est sans doute la cause de leur instabilité naturelle. Les recherches récentes visent à obtenir des cellules souches pluripotentes humaines à l'état naïf (en haut du gradient). L'importance de ces recherches dans la perspective d'applications médicales est discutée dans cette revue. <

La pluripotence est définie par la capacité d'une cellule à se différencier en tous les types cellulaires constituant un organisme adulte. Dans l'embryon au stade du blastocyste, les cellules pluripotentes constituent l'épiblaste, qui est à l'origine de tous les organes du futur individu ainsi que des tissus hématopoïétiques du sac vitellin et des tissus vasculaires de l'interface materno-fœtale [1]. Les autres constituants du blastocyste, l'endoderme primitif et le trophoblaste, sont à l'origine des annexes extra-embryonnaires. Dans le blastocyste de souris, l'épiblaste nouvellement formé n'est constitué que de 10 à 15 cellules pluripotentes. Pendant les trois jours suivant sa formation, période marquée par l'implantation de l'embryon dans la paroi utérine, les cellules pluripotentes se multiplient acti-

Vignette (Photo @ Inserm - Afsaneh Gaillard).

${ }^{1}$ Dont le lapin.

\section{Cinquante nuances de pluripotence}

Marielle Afanassieff, Irène Aksoy, Nathalie Beaujean, Pierre-Yves Bourillot, Pierre Savatier

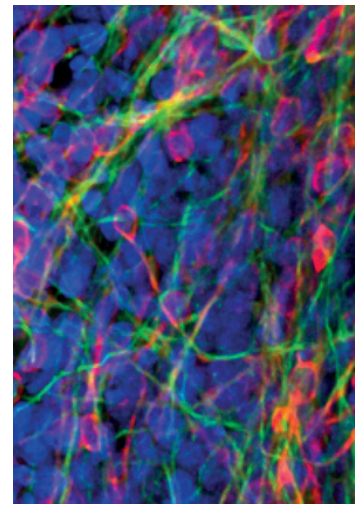

Univ Lyon, Université Lyon 1, Inserm, Stem Cell and Brain Research Institute U1208, INRA USC 1361, 69500 Bron, France pierre.savatier@inserm.fr

vement et s'organisent progressivement en une structure d'aspect épithélial. On compte 600 à 700 cellules pluripotentes dans l'épiblaste au début de la gastrulation, c'est-à-dire au début de la formation des premiers grands lignages embryonnaires: l'ectoderme, le mésoderme et l'endoderme. On en compte jusqu'à environ 10000 lorsqu'il a atteint sa pleine expansion, trois jours après l'implantation. Au-delà, toutes les cellules pluripotentes se sont engagées vers l'une de ces trois destinées embryonnaires et leur stock est alors irrémédiablement épuisé. La pluripotence est donc un état transitoire du développement embryonnaire.

Depuis les travaux précurseurs de Martin Evans, au Royaume-Uni [2], et de Gail Martin aux États-Unis en 1981 [3], il est possible de capturer cet état de pluripotence transitoire sous la forme de lignées cellulaires: les cellules souches embryonnaires pluripotentes, ou cellules ES. Contrairement aux cellules pluripotentes de l'épiblaste dont elles sont issues, les cellules ES se caractérisent par leur autorenouvellement illimité, grâce à des conditions de culture appropriées qui bloquent l'engagement dans la différenciation. Au cours des trois décennies écoulées, divers protocoles ont été élaborés dans le but d'accroître la stabilité de la pluripotence in vitro et de préserver l'intégrité génétique des cellules ainsi capturées [4]. Les lignées de cellules souches pluripotentes ainsi fabriquées ont permis des avancées considérables dans notre compréhension des mécanismes moléculaires et cellulaires qui sous-tendent la pluripotence chez la souris, et plus récemment chez l'homme. Elles ont aussi conduit à une vision plus complexe de la pluripotence. Celle-ci n'apparaît plus comme un état unique et homogène de l'épiblaste pendant toute la durée de son expansion, mais plutôt comme la succession, et parfois la coexistence, d'états discrets amenant graduellement les cellules pluripotentes aux abords de la différenciation. Dans cette revue, nous ferons le point des connaissances sur les différents états de pluripotence in vitro, ainsi 


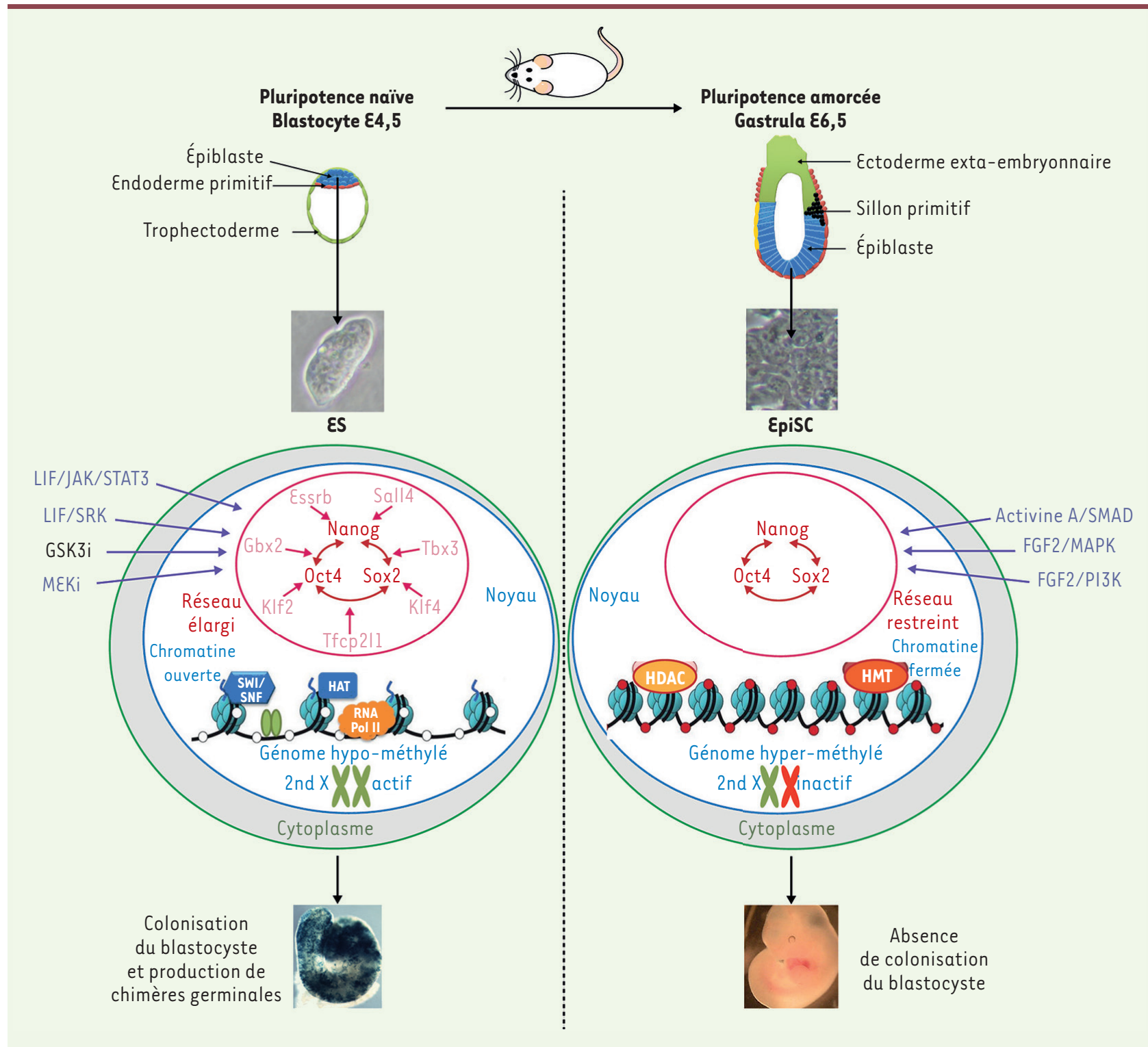

Figure 1. États naïf et amorcé de pluripotence chez la souris. $£ 4,5$ et $\varepsilon 6,5$ représentent les stades de développement embryonnaire en jours. $£ S$, embryonic stem ; EpiSC, epiblast stem cell ; LIF, leukemia inhibitory factor ; JAK, janus kinase ; STAT3, signal transducer and activator of transcription 3 ; GSK3i, inhibiteur pharmacologique de GSK3 (glycogen synthase kinase-3) ; MEKi, inhibiteur pharmacologique de MEK (mitogen-activated protein kinase kinase) ; SRK, Src-related kinases (Hck et Yes); FGF2, fibroblast growth factor 2 ; MAPK, mitogen-activated protein kinase ; PI3K, phosphoinositide 3 -kinase $; 2^{\text {nd }} \mathrm{X}$, second chromosome $\mathrm{X}$.

que sur leurs corrélats moléculaires et développementaux. Nous discuterons également des différences entre mammifères et de l'impact de ces nouvelles connaissances dans la mise en œuvre de la thérapie cellulaire.

\section{La pluripotence naïve : une tabula rasa}

La dérivation de lignées de cellules ES à partir de l'embryon préimplantatoire de souris au stade blastocyste requiert le LIF (leukemia inhibitory factor), une cytokine de la famille de I'IL(interleukine)-6, et du sérum, ce dernier pouvant être remplacé par le BMP4 (bone morphogenetic protein 4) [5] (Figure 1). Le LIF active plusieurs facteurs, dont la kinase JAK2 (Janus kinase), le facteur de transcription STAT3 (signal transducer and activator of transcription 3) [6], Ia PI3K (phosphoinositide 3-kinase) [7], et les kinases de la famille Src, Hck (hematopietic cell kinase) et Yes [8]. Le BMP4 active la transcription des gènes de la famille Id, via les facteurs de transcription de type Smad [5]. Ensemble, ils stimulent l'activité d'un réseau de gènes 
caractéristiques de l'épiblaste du blastocyste. Ce réseau comprend les facteurs de transcription centraux de la pluripotence, 0ct4 (octamerbinding transcription factor 4), Sox2 (SRY-related HMG-box gene 2) et Nanog, et plusieurs facteurs ancillaires, KIf2 (Krüppel-like factor 2), KIf4, Tfcp2Il (transcription factor CP2 like 1), Tbx3, Esrrb (estrogenrelated receptor beta), Gbx2, et Sall4 (Spalt-like 4), formant un réseau élargi $[9,10]$. Ce réseau de pluripotence peut être stabilisé et son activité renforcée, en bloquant l'activité des kinases ERK1/2 (extracellular regulated kinases $1 / 2$ ) responsables de l'engagement spontané dans la différenciation, et en stimulant l'activité de la signalisation WNT grâce à l'inhibition de la kinase GSK3 (glycogen synthase kinase 3). Dans ce but, on utilise des inhibiteurs pharmacologiques et un milieu de culture synthétique [11]. Cette condition de culture, chimiquement définie, est appelée « $2 \mathrm{i} /$ LIF $»^{1}$. Les cellules $\varepsilon S$ cultivées dans cette condition sont capables de s'auto-renouveler de manière illimitée en l'absence d'activité des protéines $\varepsilon R K$, de sérum et de BMP4. Le génome est globalement hypo-méthylé, la chromatine est dans une configuration permissive, et les cellules des lignées dérivées d'embryons femelles possèdent deux chromosomes $X$ actifs, autant de caractéristiques des cellules pluripotentes du blastocyste. L'ensemble de ces caractéristiques définit l'état naïf, ou état fondamental de pluripotence. Sur le plan fonctionnel, l'état naï se distingue par la capacité de chaque cellule $\varepsilon S$ de souris, une fois réintroduite dans un blastocyste hôte, à s'incorporer dans l'épiblaste et à participer ainsi à la formation de tous les tissus constituant l'organisme adulte, y compris la lignée germinale [12, 13]. L'entité ainsi obtenue est appelée « chimère germinale » car tous ses tissus, y compris les gamètes, sont constitués d'un mélange de cellules différenciées dérivant des cellules pluripotentes de l'embryon hôte et de cellules différenciées dérivant des cellules ES introduites expérimentalement dans l'embryon. Les analyses par séquençage de l'ARN (RNA-seq) ont montré que le transcriptome des cellules $E S$ est très proche de celui de l'épiblaste du blastocyste entre la fin du $4^{\mathrm{e}}$ et le $5^{\mathrm{e}}$ jour de développement [14]. Ce stade du développement est d'ailleurs celui à partir duquel des lignées de cellules ES de souris peuvent être dérivées avec la plus grande efficacité. À partir du $6^{\mathrm{e}}$ jour, cette efficacité chute brutalement. Les cellules $\varepsilon S$ de souris en culture peuvent également se différencier en gamètes mâles et femelles fonctionnelles [15], ce qui est cohérent avec le fait que la ségrégation de la lignée germinale ne s'opère qu'au $6^{\mathrm{e}}$ jour de développement chez la souris. Ainsi, la combinaison $2 \mathrm{i} / \mathrm{LIF}$ permet de capturer in vitro, avec une fidélité quasi parfaite, l'état de pluripotence qui prévaut dans l'épiblaste de l'embryon au début du $5^{\mathrm{e}}$ jour de son développement.

Le rôle de la signalisation du LIF dans la capture de l'état naïf de pluripotence mérite attention. Les études génétiques ont révélé qu'aucun des composants de la voie de signalisation du LIF (son récepteur LIFR, son co-récepteur, la glycoprotéine gp 130, et STAT3) n'était nécessaire au développement de l'embryon pré-implantatoire. L'activation de la voie LIF/STAT3 se révèle néanmoins nécessaire à la survie du blastocyste lors de la diapause, un processus physiologique qui, chez les

${ }^{1}$ Voir Glossaire. rongeurs, retarde l'implantation chez la femelle allaitante. Dans cette situation seulement, la voie LIF/STAT3 maintient les cellules de l'épiblaste à l'état pluripotent [16]. Son activation serait donc la solution adoptée par la future cellule ES afin d'acquérir l'immortalité.

\section{La pluripotence amorcée : un état fragile à la lisière de la différenciation}

Il est impossible de dériver des lignées de cellules \&S à partir de l'épiblaste de l'embryon de souris au-delà du $5^{e}$ jour de développement [17]. Néanmoins, les cellules de l'épiblaste sont encore pluripotentes, et ce jusqu'au début du $9^{\mathrm{e}}$ jour. À partir du $6^{\mathrm{e}}$ jour, les cellules de l'épiblaste ont simplement perdu la capacité de répondre au LIF. En revanche, elles ont acquis la capacité de s'autorenouveler in vitro sous l'action du FGF2 (fibroblast growth factor 2) et de l'Activine A (ActA). Les lignées de cellules ainsi obtenues sont appelées EpiSC (epiblast stem cells) [18, 19]. Les cellules EpiSC sont capables de se différencier in vitro en cellules représentatives des trois feuillets embryonnaires, attestant de la préservation de leur caractère pluripotent. Contrairement au milieu de culture des cellules ES, celui des EpiSC est enrichi en facteurs sériques, reflétant la relative complexité des voies de signalisation impliquées dans le contrôle de leur auto-renouvellement. Le transcriptome des cellules EpiSC est très proche de celui de l'épiblaste de l'embryon post-implantatoire dont elles sont issues et diffère de celui des cellules ES [18, 19]. Singulièrement, les cellules EpiSC ont un transcriptome similaire à celui de l'épiblaste de l'embryon au stade de la gastrula tardive (correspondant au $8^{\text {e }}$ jour de développement), et ce quel que soit le stade embryonnaire (entre le $6^{\mathrm{e}}$ et le $8^{e}$ jour) utilisé comme source de l'épiblaste [20] (Figure 1). Les voies de signalisation FGF2 et Activine A ont donc la capacité de conduire les cellules de l'épiblaste vers un état unique, temporellement restreint, propice à leur auto-renouvellement. Une particularité du transcriptome des cellules EpiSC est la présence d'ARN messagers (ARNm) caractéristiques des tous premiers stades de la différenciation en ectoderme (Fgf5 [fibroblast growth factor 5], Sox1), en mésoderme (Brachyury) et en endoderme (Foxa2 [forkhead box protein A2], Sox17, Cer1 [Cereberus]), qui conduit à l'idée que les cellules EpiSC, bien que fonctionnellement pluripotentes, sont pré-instruites pour la différenciation. La notion de pluripotence amorcée a été proposée pour rendre compte de l'état des cellules EpiSC, un état plus proche de l'engagement dans la différenciation que l'état naïf [13]. Les cellules EpiSC expriment les régulateurs canoniques de la pluripotence, 0 ct4, Sox2 


\section{GLOSSAIRE}

ES : cellule souche embryonnaire

LIF : leukemia inhibitory factor

IL : interleukine

BMP : bone morphogenetic protein

JAK : janus kinase

STAT3 : signal transducer and activator of transcription 3

PI3K : phosphoinositide 3-kinase

Hck : hematopoietic cell kinase

0ct4 : octamer-binding transcription factor 4

Sox2 : SRY-related HMG-box gen 2

KIf : Krüppel-like factor

Tfcp211 : transcription factor CP2-like 1

Esrrb : estrogen-related receptor beta

Sall4 : Splat-like 4

ERK : extracellular regulated kinase

GSK3 : glycogen synthase kinase 3

LIFR : récepteur du LIF

gp130 : co-récepteur du LIF

FGF2,4,5 : fibroblast growth factor 2,4,5

Foxa2 : forkhead box protein A2

Cerl : Cereberus

rsEpiSC : region-specific epiblast stem cells

INTPSC : intermediate pluripotent stem cell

عPL : primitive ectoderm-like cell

IESC : intermediate embryonic stem cell

Otx2 : orthodenticle homeobox 2

Lef1: lymphoid enhancer binding factor 1

ROCK : Rho-associated protein kinase

HNES : human naïve embryonic stem

MAPK : mitogen-activated protein kinase

MEK : mitogen-activated protein kinase kinase

PKA : protein kinase $A$

PKC : protein kinase $C$

JNK : c-Jun N-terminal kinase

HDAC : histone deacetylase

HPAT5 : human pluripotency-associated transcripts 5

TFAP2C : transcription factor AP-2 gamma

$2 \mathbf{i}$ : inhibiteur de GSK3 + inhibiteur de MEK mation des trois feuillets embryonnaires [20, 23]. On ne sait pas si elles sont capables de former tous les tissus différenciés de l'organisme adulte car ces expériences ont été réalisées sur des embryons post-implantatoires cultivés in vitro pendant 48 heures, au-delà desquelles ils dégénèrent. En revanche, contrairement aux cellules $\varepsilon S$, les cellules EpiSC sont incapables de coloniser l'épiblaste de l'embryon pré-implantatoire et former des chimères germinales. Elles ont également perdu la capacité de se différencier en gamètes, ce qui est cohérent avec la ségrégation de la lignée germinale lors $\mathrm{du} 6^{\mathrm{e}}$ jour de gestation chez la souris.

Une nouvelle classe de lignées EpiSC, appelée rsEpiSC (region-selective epiblast stem cells), a été dérivée de l'épiblaste d'embryons au stade gastrula ( $8^{\text {e }}$ jour de développement) en utilisant un inhibiteur de la signalisation WNT, la molécule IWRI ${ }^{2}$ [24]. Les cellules rsepiSC expriment des marqueurs transcriptomiques caractéristiques de la région postérieure de l'épiblaste. De plus, elles colonisent préférentiellement cette région lorsqu'elles sont réintroduites dans l'embryon au stade gastrula, alors que les cellules EpisC conventionnelles présentent les caractéristiques transcriptomiques et fonctionnelles propres à la région antérieure de l'épiblaste [20]. La dérivation des lignées de cellules EpiSC conduit donc à une restriction spatiale des caractéristiques des cellules souches pluripotentes.

Pour conclure, la combinaison FGF2/Activine A permet de capturer in vitro, avec une fidélité quasi parfaite, l'état de pluripotence qui prévaut dans les dernières cellules pluripotentes de l'embryon, et plus particulièrement des cellules localisées dans la partie antérieure ou la partie postérieure de l'épiblaste de la gastrula tardive, à la lisière de la différenciation.

\section{Vers une modélisation du continuum de pluripotence}

Les états naïfs et amorcés incarnent les deux extrémités d'un continuum de pluripotence allant de l'épiblaste précoce du blastocyste à l'épiblaste tardif de la gastrula. Grâce aux cellules ES et EpiSC, il devient possible de modéliser ce continuum in vitro. Les cellules $\varepsilon S$ peuvent être facilement converties en cellules de type EpiSC en les cultivant dans un milieu contenant les facteurs de remplacement sérique, le FGF2 et l'Activine A [22]. La transition s'accompagne de l'acquisition par les cellules de leur dépendance aux voies de signalisation FGF2/ERK et Activine A/Smad, de la disparition des

${ }^{2} 4$-(1,3,3a, 4,7, 7a-Hexahydro-1,3-dioxo-4,7-methano-2H-isoindol-2-yl)-N8 -quinolinyl-Benzamide. 


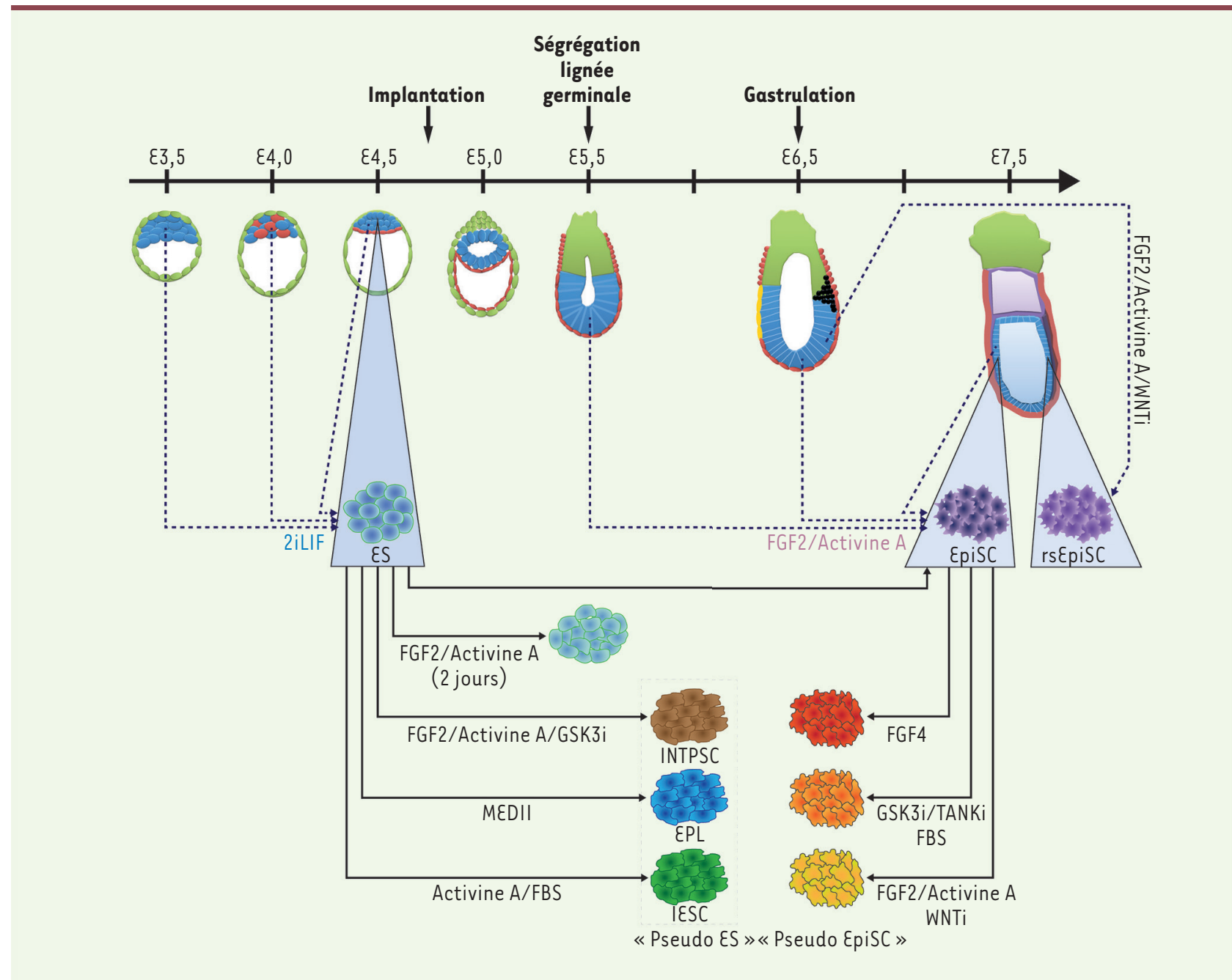

Figure 2. Le continuum de pluripotence chez la souris. $\varepsilon 3,5$ à $\varepsilon 7,5$ représentent les stades de développement embryonnaire en jours. Les flèches pointillées indiquent la dérivation de lignées de cellules souches pluripotentes à partir de l'épiblaste embryonnaire à différents stades de son développement. Les triangles bleus indiquent la correspondance entre cellules souches pluripotentes et stades embryonnaires. Les flèches pleines indiquent: (i) la conversion antérograde des cellules ES en cellules plus immatures appelées EpiSC (epiblast stem cell), EpiLC (epiblast-like cell), INTPSC (intermediate pluripotent stem cell), EPL (primitive ectoderm-like cell), et IESC (intermediate embryonic stem cell); (ii) la conversion rétrograde des cellules EpiSC. FBS : sérum de bœuf fœtal ; GSK3i : inhibiteur pharmacologique de la kinase GSK3 (glycogen synthase 3 beta); MEDII : milieu conditionné de la lignée HepG2 ; rsEpiSC : region-specific epiblast stem cell ; TANKi : inhibiteur pharmacologique de la tankyrase ; WNTi : inhibiteur pharmacologique de la voie de signalisation WNT; 2iLIF : GSK3i (inhibiteur de la glycogen synthase 3 beta) + MEKi (inhibiteur de la mitogen-activated protein kinase kinase) + LIF (leukemia inhibitory factor).

marqueurs transcriptomiques de l'épiblaste précoce et de l'acquisition des marqueurs de l'épiblaste tardif [25]. La question de l'identification d'éventuels états intermédiaires susceptibles de modéliser l'épiblaste entre le $5^{\mathrm{e}}$ et le $8^{\mathrm{e}}$ jour de développement est plus difficile. Néanmoins, quelques travaux apportent des premiers éléments de réponse (Figure 2). Plusieurs méthodes permettant de sortir les cellules ES hors de leur état naïf originel ont été décrites. Par exemple, le traitement des cellules ES par le FGF2 et l'Activine A pendant 2 jours, en présence d'une faible concentration de facteurs de remplacement sérique, amorce la différenciation et conduit à la formation de cellules appelées EpiLC (epiblast-like cells) [26]. Ces cellules ont un transcriptome similaire à celui de l'épiblaste de l'embryon post-implantatoire avant la gastrulation ( $6^{\mathrm{e}}$ jour de développement) mais différent de celui des cellules EpiSC. Comme les cellules ES, et contrairement aux cellules EpiSC, elles sont capables de se différencier dans la lignée germinale. Les cellules EpilC semblent donc occuper une position intermédiaire 
entre état naïf et état amorcé, tant sur le plan transcriptomique que sur le plan fonctionnel. Un autre protocole utilise le FGF2, l'Activine A et un inhibiteur chimique de la kinase GSK3 afin de convertir les cellules \&S naïves en cellules appelées INTPSC (intermediate pluripotent stem cells), un état décrit comme intermédiaire entre état naïf et état amorcé sur la base de l'analyse du transcriptome [27]. Les cellules INTPSC sont capables de produire des chimères germinales après injection dans un blastocyste, suggérant une proximité fonctionnelle avec les cellules EpiLC. L'inactivation de la signalisation WNT semble aider les cellules EpiSC à sortir de l'état amorcé, à acquérir les caractéristiques transcriptomiques d'un épiblaste plus immature, distinctives de l'embryon avant le début de la gastrulation, mais néanmoins différentes de celles des cellules $\varepsilon S[28,29]$. Le FGF4 exercerait un effet similaire en permettant aux cellules EpiSC de coloniser le blastocyste, comme le font les cellules $\varepsilon S$, sans néanmoins contribuer à la formation de la lignée germinale [30]. D'autres types cellulaires ont été créés à partir de cellules $\varepsilon S$, comme les cellules EPL (primitive ectoderm-like cell) et les cellules IESC (intermediate embryonic stem cell), mais leur relevance pour la modélisation du continuum de pluripotence est moins claire. Enfin, un nouvel état dit formatif, situé entre l'état naïf et l'état amorcé, a récemment été proposé [31]. À l'état formatif, les cellules pluripotentes n'expriment plus les gènes du noyau de la pluripotence naïve (Klf2, Klf4, Tfcp2l1, Tbx3, Esrrb, Gbx2, et Sall4) et pas encore les gènes de l'ectoderme (Fgf5, Soxl), du mésoderme (Brachyury) et de l'endoderme (Foxa2, Cer1, Sox17). Elles expriment d'autres régulateurs présomptifs de la pluripotence tels que Oct6 (Pou3f1), Otx2 (orthodenticle homeobox 2) et Lefl (lymphoid enhancer binding factor 1), dont l'expression est également observée dans l'épiblaste au $6^{\mathrm{e}}$ jour de développement, c'est-à-dire au moment de la ségrégation de la lignée germinale. L'état formatif représenterait un état intermédiaire au sein du continuum de pluripotence pendant lequel les cellules ne constituent plus une tabula rasa (cellules ES), mais ne sont pas encore des cellules pré-instruites à se différencier en l'un des trois feuillets embryonnaires (cellules EpiSC). Cette phase intermédiaire permettrait de déclencher le reformatage du transcriptome, de l'épigénome, et des réseaux de signalisation, conférant ainsi aux cellules pluripotentes la compétence pour répondre aux signaux de différenciation lors de la gastrulation [32].

Ces travaux révèlent divers états intermédiaires de pluripotence dans les cultures cellulaires. Tous ne sont cependant pas des états stables. Les cellules EpiLC, par exemple, n'existent que de manière transitoire sur le chemin menant les cellule $\varepsilon S$ vers la différenciation germinale [26]. L'état formatif est, lui aussi, observé de manière transitoire $16 \mathrm{~h}$ après la sortie des cellules ES de l'état naïf. On ne connaît pas encore les voies de signalisation dont l'activation permettrait de stabiliser ces états fugaces. Une autre difficulté est la coexistence de différents états au sein d'une population cellulaire a priori homogène, un phénomène observé aussi bien dans les populations de cellules $E S$ que de cellules EpiSC [33]. Par ailleurs, aucun de ces protocoles n'est encore capable de simuler l'intégralité de l'évolution de la pluripotence partant de la tabula rasa (cellules ES) jusqu'à la lisière de la différenciation (cellules Episc).

\section{Les cellules « $\varepsilon S$ » et le continuum de pluripotence chez l'homme}

Les premières tentatives visant à créer des lignées de cellules ES naïves à partir de blastocystes humains en utilisant les protocoles développés chez la souris ont échoué, suggérant que, chez l'homme, le LIF est incapable d'enclencher le processus d'immortalisation des cellules de l'épiblaste et que l'inhibition de ERK n'est pas suffisante pour bloquer leur différenciation. Néanmoins, des lignées de cellules souches pluripotentes humaines ont été obtenues en utilisant le FGF2 (ou la combinaison FGF2/Activine A) et des facteurs de remplacement sérique $[34,35]$. Les lignées ainsi obtenues sont souvent appelées «ES » mais elles ne présentent en réalité aucune des caractéristiques cardinales de la pluripotence naïve telles que définies chez la souris. Outre l'absence de réponse à la signalisation LIF/STAT3 [36], elles requièrent l'activité des kinases ERK pour se diviser, elles n'expriment aucun des facteurs de pluripotence ancillaires décrits chez la souris [37, 38], la respiration mitochondriale est réduite [39], le génome est hyper-méthylé [37], la chromatine est enrichie en marques répressives des histones [40] et elles ne possèdent qu'un seul chromosome $X$ actif dans les lignées dérivées d'embryons humains XX [37]. La comparaison de leur transcriptome à celui de l'épiblaste de singe macaque cynomolgus (ou macaque crabier), entre le $6^{\mathrm{e}}$ (blastocyste) et le $17^{\mathrm{e}}$ jour de développement (gastrula), montre que les cellules « $\varepsilon S$ » humaines ont un profil d'expression génique plus proche de celui de l'épiblaste tardif de la gastrula que de l'épiblaste précoce du blastocyste [41]. Enfin, lorsqu'elles sont injectées dans des embryons de souris au $8^{e}$ jour de développement, pour former des chimères dites «inter-espèces », elles colonisent l'épiblaste tardif de l'embryon [42] comme le font les cellules EpiSC de souris [23]. En revanche, lorsqu'elles sont injectées dans des blastocystes de souris, elles sont alors incapables de coloniser l'épiblaste précoce comme le feraient les cellules ES naïves [43]. Ainsi, selon tous les critères communément utilisés pour positionner les cellules le long du continuum de pluripotence, les cellules «ES » humaines s'auto-renouvellent dans un état de pluripotence proche, voire identique à celui de l'état amorcé décrit pour les cellules EpiSC de souris (Figure 3). L'usage des guillemets pour décrire ces cellules « $\varepsilon S$ » s'impose donc afin de les distinguer des véritables cellules $E S$ à l'état naïf.

La raison pour laquelle les cellules « $\varepsilon S$ » humaines ne s'auto-renouvellent pas à l'état naïf est une question fondamentale. Selon I'hypothèse la plus couramment proposée, l'absence de diapause aurait conduit au 


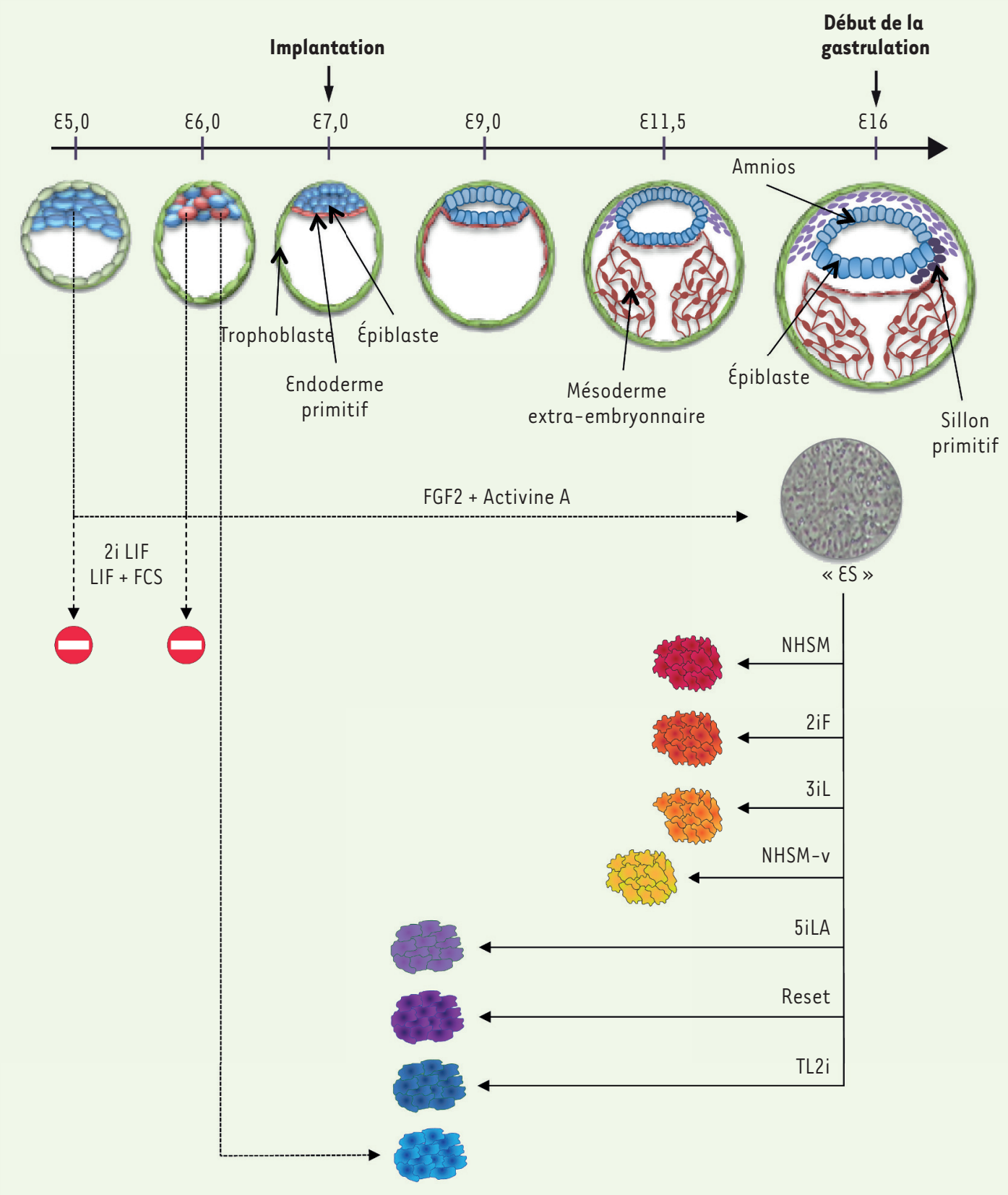

Figure 3. Le continuum de pluripotence chez l'homme. $\varepsilon 5$ à $\varepsilon 16$ représentent les stades de développement (en jours). Les flèches pointillées indiquent la dérivation de lignées de cellules souches pluripotentes à partir de l'épiblaste embryonnaire à différents stades de son développement. Les flèches pleines indiquent la conversion rétrograde des cellules « $\varepsilon S$ » humaines en cellules plus immatures, appelées NHSM (naive human stem medium), 3i, 2iF, NHSM-v (naive human stem medium + vitamine C), 5iLA, Reset, et TL2i. HNES : human naive embryonic stem); FCS : sérum de veau fœtal ; 2i : GSK3i (inhibiteur de la glycogen synthase 3 beta) + MEKi (inhibiteur de la mitogen-activated protein kinase kinase) ; LIF : leukemia inhibitory factor.

démantèlement de la voie de signalisation du LIF chez les primates. Ainsi, lorsqu'elles sont mises en culture, les cellules de l'épiblaste précoce humain continuent de progresser spontanément le long du continuum de pluripotence jusqu'à atteindre un état amorcé où elles se stabilise sous l'action du FGF2 et de l'Activine A. Cette dérive spontanée des cellules de l'épiblaste naïf vers l'état amorcé s'observe d'ailleurs aussi chez la souris, lorsque l'épiblaste précoce du blastocyste est 
cultivé en l'absence de LIF et en présence de FGF2 et d'Activine A. On obtient alors des cellules EpiSC, même si l'épiblaste originel était de nature intrinsèquement naïve.

La situation que nous venons de décrire chez l'homme n'a rien d'exceptionnelle. Au contraire, les cellules «ES» dérivées de blastocystes chez d'autres primates (macaque, marmouset, babouin), mais aussi chez le lapin, le vison, le chien et le bovin, présentent les caractéristiques cardinales de l'état amorcé $[4,41]$. La souris fait donc figure d'exception, soulignant la nécessité d'étudier le continuum de pluripotence dans les embryons de primates, humains et non-humains.

\section{Comment obtenir des cellules ES humaines naïve ?}

En 2016, l'équipe de Austin Smith, à Cambridge (Royaume-Uni), a réussi à obtenir des lignées de cellules $E S$ humaines dans un état proche de l'état naïf en utilisant un milieu de culture similaire à celui utilisé pour la culture des cellules ES de souris, mais dans lequel ont été ajoutés des inhibiteurs de la protéine kinase C (le Gö6983) et de la kinase ROCK (Rho-associated protein kinase, le Y-27632), ainsi que de la vitamine C (milieu t2iLGöy). Les lignées de cellules ainsi obtenues, appelées HNES (human naive embryonic stem), expriment les facteurs de transcription accessoires spécifiques de la pluripotence naïve telle que définie chez la souris. Elles présentent un profil global d'expression génique et un niveau de méthylation du génome différents de celui des cellules « $\varepsilon S$ » dérivées en milieu FGF2/Activine A, et compatibles avec des caractéristiques de pluripotence naïve [44]. Les cellules HNES ne sont pour autant pas de véritables cellules ES naïves car elles sont incapables de coloniser l'embryon animal pour former des chimères, une marque des véritables cellules ES naïves chez le souris. D'ailleurs, les analyses à l'échelle unicellulaire ont révélé que le transcriptome des cellules HNES est sensiblement différent de celui des cellules de l'épiblaste précoce dont elles sont issues, laissant penser que le système de culture t2iLGöy est capable de bloquer la dérive des cellules en direction de la pluripotence amorcée, mais ne réussit pas à véritablement capturer l'état de pluripotence qui caractérise les cellules de l'épiblaste du blastocyste humain (Figure 3).

Une étude systématique des facteurs de croissance, cytokines et molécules capables de promouvoir l'immortalisation des cellules de l'épiblaste précoce et la dérivation de véritables lignées de cellules ES humaines naïves est donc nécessaire. La difficulté d'accès aux embryons humains rend cependant cette approche difficile. Une autre approche consiste à reprogrammer les cellules «ES» humaines conventionnelles en cellules exprimant les caractéristiques épigénétiques et fonctionnelles de cellules $E S$ naïves. Plusieurs protocoles ont été ainsi élaborés visant à repousser les cellules « $E S$ » de primates, humain et non-humain, vers le haut du continuum de pluripotence, en utilisant des facteurs de transcription associés à la pluripotence naïve chez la souris (OCT4, KLF2, KLF4, NANOG et STAT3), des facteurs de croissance (LIF, Activine A, FGF2), ainsi que des inhibiteurs de kinases (ROCK, BRAF, p38/MAPK [mitogen-activated protein kinase], GSK3, MEK [mitogen-activated protein kinase kinase] 1 \& 2, SRC, PKA (protein kinase A), PKC (protein kinase C), RAF et JNK [c-Jun $N$-terminal kinase]), d'histones déacétylases ( $\mathrm{HDACl} / 2$ ), ou de voies de signalisation (BMP et HIPPO). Les cellules ainsi obtenues présentent des caractéristiques de l'état naïf de pluripotence, tel qu'il est défini chez la souris, avec une reconfiguration du transcriptome et de l'épigénome les rapprochant de l'épiblaste précoce du blastocyste $[38,41,45]$. On observe également la perte de la dépendance à la signalisation FGF2/ERK, I'acquisition de la dépendance à la signalisation LIF/JAK/STAT3, la stimulation de la respiration mitochondriale, et la réactivation du second chromosome $X$. Notons cependant qu'aucune des lignées de cellules $\varepsilon S$ humaines ainsi reprogrammées ne présente toutes les caractéristiques de l'état naïf de pluripotence tel qu'il est défini chez la souris [46-49]. Par ailleurs, aucune de ces cellules ne semble capable de former des chimères après injection dans un embryon animal, une prérogative des véritables cellules ES naïves. La véritable cellule ES humaine naïve semble donc ne pas encore exister.

\section{Conclusions}

Quelle est la signification de cette pléthore de lignées? Il est possible que les cellules de ces lignées se positionnent à différents niveaux le long du continuum de pluripotence, mais qu'aucune n'ait encore atteint l'état naïf tel qu'il est défini chez la souris. II est également possible que certaines de ces lignées représentent des artéfacts, sans correspondance avec un quelconque stade de développement de l'épiblaste humain. Singulièrement, toutes ces lignées ont en commun la réactivation de la signalisation LIF/JAK/STAT3 et la perte de la dépendance à la voie MEK/ERK qui sont les premiers critères moléculaires de l'état naïf chez la souris, alors qu'il n'existe aucune preuve tangible de l'implication de ces voies dans le développement du blastocyste humain. Il semble donc vraisemblable que les mécanismes moléculaires qui contrôlent l'émergence et la multiplication des cellules pluripotentes dans le blastocyste humain soient partiellement différents de ceux décrits chez les rongeurs. Un long ARN non codant, HPAT5 (human pluripotency-associated transcripts 5 ), ainsi qu'un facteur de transcription, TFAP2C (transcription factor AP-2 gamma), spécifiques de l'état naïf de la pluripotence humaine ont déjà été identifiés. La capture de l'état naïf dans les cellules souches pluripotentes humaines requiert maintenant l'identification des voies de signalisations qui régulent cet état dans l'embryon de primates [50]. Les études récentes du transcriptome de l'embryon humain ouvrent la voie à cette connaissance [51].

La dichotomie entre cellules $E S$ naïves et cellules «ES » amorcées s'applique-t-elle aussi aux cellules souches 
pluripotentes induites (iPS) ? Toutes les études montrent que les cellules iPS obtenues chez les rongeurs s'auto-renouvellent à l'état naïf alors que les cellules iPS obtenues chez les primates s'auto-renouvellent à l'état amorcé. Parmi tous les protocoles développés afin de reprogrammer les cellules « $E S$ » humaines vers l'état naïf, certains ont été appliqués avec succès aux cellules iPS. II est donc vraisemblable que les résultats des futures recherches sur les cellules ES naïves humaines pourront être appliqués à l'obtention de cellules iPS naïves. Pourquoi est-il important d'obtenir des cellules ES et iPS naïves chez l'homme? Les cellules souches pluripotentes à l'état amorcé sont beaucoup plus instables que les cellules naïves tant sur le plan de l'intégrité génétique que celui de l'auto-renouvellement. Contrairement à la culture des cellules ES de souris, celle des cellules «ES » et iPS humaines requiert des milieux de culture sophistiqués et un contrôle qualité régulier pour s'assurer de l'absence de dérives génétiques dans les populations cellulaires. Des conditions de culture non optimales sont d'ailleurs souvent à l'origine de l'accumulation de cellules possédant des réarrangements chromosomiques car les altérations ainsi produites leur confèrent un avantage de croissance. Elles subissent alors une sélection positive darwinienne [52], constituant un risque pour les applications en thérapie cellulaire [53]. Des différences dans les capacités de différenciation ont également été décrites entre des lignées de cellules «ES » et des lignées de cellules iPS, différences qui pourraient s'expliquer par une plus grande hétérogénéité épigénétique. Toutes ces «faiblesses » tiennent sans doute au fait que les cellules pluripotentes amorcées sont plus proches de l'engagement dans la différenciation. Contrairement aux cellules naïves dont la fonction première, dans l'embryon, est uniquement l'amplification rapide du stock de cellules pluripotentes avant l'implantation, les cellules amorcées sont dans une situation d'équilibre précaire car elles sont programmées pour répondre rapidement aux signaux de différenciation lors de la gastrulation. C'est d'ailleurs la raison d'être du continuum de pluripotence : celle de permettre aux cellules pluripotentes d'évoluer graduellement d'une phase de multiplication rapide (phase naïve) à une phase d'engagement imminent dans la différenciation (phase amorcée). Il est donc vraisemblable que l'instabilité décrite chez les cellules « ES » humaines soit consubstantielle de l'état amorcé. L'obtention de cellules ES et iPS humaines naïves permettra de s'affranchir de cette instabilité et facilitera la manipulation des cellules souches pluripotentes à finalité thérapeutique.

Seules les cellules ES et iPS naïves possèdent la capacité de coloniser l'embryon et de former des chimères. Cette compétence est le fondement de la technologie utilisée pour l'obtention des souris KO (knockout). Chez l'homme, l'obtention de cellules ES et iPS naïves est une condition préalable à la mise en œuvre d'une nouvelle technologie visant à produire des organes humains chez l'animal $[54,55]$. Cette technologie, qui en est encore à ses balbutiements, repose sur l'injection de cellules \&S ou iPS humaines dans l'embryon d'un animal (porc, mouton) dans lequel un ou plusieurs gènes « maîtres » de la formation des organes ont été invalidés. Les cellules humaines ainsi injectées pourraient alors complémenter la fonction manquante et créer un organe entièrement humain chez l'animal devenu adulte. Sa mise en œuvre exigera de disposer de cellules humaines, ES ou iPS, à l'état naïf de pluripotence afin de coloniser efficacement l'embryon animal. $\diamond$

\section{SUMMARY}

\section{Fifty shades of pluripotency}

Since the derivation of the first pluripotent embryonic stem cell lines in mice in the early 1980s, a plethora of lines has been obtained from various mammalian species including rodents, lagomorphs and primates. These lines are distinguished by their molecular and functional characteristics and correspond to the different pluripotency states observed in the developing embryo between the "blastocyst" and "gastrula" stages. These cell lines are positioned along a gradient, or continuum of pluripotency, the ends of which are epitomized by the naïve and primed states, respectively. Conventional human pluripotent stem cells self-renew in the primed state of pluripotency (ie, at the bottom of the gradient), a position that is undoubtedly the cause of their natural instability. Recent studies aim to generate naive human pluripotent stem cells (at the top of the gradient). The importance of this research in the perspective of medical applications will be discussed. $\diamond$

\section{REMERCIEMENTS}

Infrastructure Nationale en Biologie et Santé INGESTEM (ANR-11INBS-0009), IHU-B CESAME (ANR-10-IBHU-003), LabEx DEVweCAN (ANR-10-LABX-0061), LabEX CORTEX (ANR-11-LABX-0042).

\section{LIENS D'INTÉRÊT}

Les auteurs déclarent n'avoir aucun lien d'intérêt concernant les données publiées dans cet article.

\section{RÉFÉRENCES}

1. Gardner RL, Rossant J. Investigation of the fate of 4-5 day post-coitum mouse inner cell mass cells by blastocyst injection. J Embryol Exp Morphol $1979 ; 52: 141-52$.

2. Evans MJ, Kaufman MH. Establishment in culture of pluripotential cells from mouse embryos. Nature $1981 ; 292$ : 154-6.

3. Martin GR. Isolation of a pluripotent cell line from early mouse embryos cultured in medium conditioned by teratocarcinoma stem cells. Proc Natl Acad Sci U SA $1981 ; 78$ : 7634-8.

4. Savatier P, Osteil P, Tam PP. Pluripotency of embryo-derived stem cells from rodents, lagomorphs, and primates: Slippery slope, terrace and cliff. Stem Cell Res 2017 ; 19 : 104-12.

5. Ying QL, Nichols J, Chambers I, Smith A. BMP induction of Id proteins suppresses differentiation and sustains embryonic stem cell self-renewal in collaboration with STAT3. Cell $2003 ; 115: 281-92$.

6. Niwa H, Burdon T, Chambers I, Smith A. Self-renewal of pluripotent embryonic stem cells is mediated via activation of STAT3. Genes Dev 1998 ; $12: 2048-60$.

7. Paling NR, Wheadon H, Bone HK, Welham MJ. Regulation of embryonic stem cell self-renewal by phosphoinositide 3-kinase-dependent signalling. J Biol Chem $2004 ; 279:$ 48063-70.

8. Tamm C, Bower N, Anneren C. Regulation of mouse embryonic stem cell selfrenewal by a Yes-YAP-TEAD2 signaling pathway downstream of LIF. J Cell Sci $2011 ; 124: 1136-44$

9. Martello G, Smith A. The nature of embryonic stem cells. Annu Rev Cell Dev Biol 2014 ; 30 : 647-75. 


\section{RÉFÉRENCES}

10. Dunn SJ, Martello G, Yordanov B, et al. Defining an essential transcription factor program for naive pluripotency. Science 2014 ; 344 : 1156-60.

11. Ying QL, Wray J, Nichols J, et al. The ground state of embryonic stem cell self-renewal. Nature $2008 ; 453: 519-23$.

12. Beddington RS, Robertson $\varepsilon$ J. An assessment of the developmental potential of embryonic stem cells in the midgestation mouse embryo. Development $1989 ; 105: 733-7$.

13. Nichols J, Smith A. Naive and primed pluripotent states. Cell Stem Cell 2009; $4: 487-92$.

14. Boroviak $T$, Loos R, Bertone $P$, et al. The ability of inner-cell-mass cells to self-renew as embryonic stem cells is acquired following epiblast specification. Nat Cell Biol $2014 ; 16: 516-28$.

15. Saitou M, Miyauchi H. Gametogenesis from Pluripotent Stem Cells. Cell Stem Cell 2016; 18 : $721-$ 35.

16. Nichols J, Chambers I, Taga T, Smith A. Physiological rationale for responsiveness of mouse embryonic stem cells to gp 130 cytokines. Development $2001 ; 128: 2333-9$.

17. Brook FA, Gardner RL. The origin and efficient derivation of embryonic stem cells in the mouse. Proc Natl Acad Sci U SA 1997 ; 94 : 5709-12.

18. Brons IG, Smithers LE, Trotter MW, et al. Derivation of pluripotent epiblast stem cells from mammalian embryos. Nature 2007 ; 448 : 191-5.

19. Tesar PJ, Chenoweth JG, Brook FA, et al. New cell lines from mouse epiblast share defining features with human embryonic stem cells. Nature $2007 ; 448$ : 196-9.

20. Kojima Y, Kaufman-Francis K, Studdert JB, et al. The transcriptional and functional properties of mouse epiblast stem cells resemble the anterior primitive streak. Cell Stem Cell $2013 ; 14: 107-20$.

21. Ficz G, Hore TA, Santos F, et al. FGF signaling inhibition in ESCs drives rapid genome-wide demethylation to the epigenetic ground state of pluripotency. Cell Stem Cell $2013 ; 13: 351-9$

22. Guo G, Yang J, Nichols J, et al. Klf4 reverts developmentally programmed restriction of ground state pluripotency. Development $2009 ; 136: 1063-9$.

23. Huang $Y$, Osorno R, Tsakiridis A, Wilson V. In vivo differentiation potential of epiblast stem cells revealed by chimeric embryo formation. Cell reports $2012 ; 2$ : 1571-8.

24. Wu J, Okamura D, Li M, et al. An alternative pluripotent state confers interspecies chimaeric competency. Nature $2015 ; 521: 316-21$

25. Osteil P, Studdert J, Wilkie $\varepsilon$, et al. Generation of genome-edited mouse epiblast stem cells via a detour through ES cell-chimeras. Differentiation 2016 ; 91 : 119-25.

26. Hayashi $\mathrm{K}$, Ohta $\mathrm{H}$, Kurimoto $\mathrm{K}$, et al. Reconstitution of the mouse germ cell specification pathway in culture by pluripotent stem cells. Cell $2011 ; 146: 519-32$.

27. Tsukiyama T, Ohinata Y. A modified EpiSC culture condition containing a GSK3 inhibitor can support germline-competent pluripotency in mice. PLoS ONE 2014 ; 9 : e95329.

28. $\mathrm{Kim} \mathrm{H}, \mathrm{Wu}$ J, Ye S, et al. Modulation of beta-catenin function maintains mouse epiblast stem cell and human embryonic stem cell self-renewal. Nat Commun $2013 ; 4: 2403$.

29. Kurek D, Neagu A, Tastemel M, et al. Endogenous WNT signals mediate BMP-induced and spontaneous differentiation of epiblast stem cells and human embryonic stem cells. Stem cell reports $2015 ; 4: 114-28$.

30. Joo JY, Choi HW, Kim MJ, et al. Establishment of a primed pluripotent epiblast stem cell in FGF4based conditions. Sci Rep $2014 ; 4: 7477$.

31. Kalkan T, Olova N, Roode M, et al. Tracking the embryonic stem cell transition from ground state pluripotency. Development 2017 ; 144 : 1221-34.

32. Smith A. Formative pluripotency: the executive phase in a developmental continuum. Development 2017 ; 144 : 365-73.

33. Han DW, Tapia N, Joo Jy, et al. Epiblast stem cell subpopulations represent mouse embryos of distinct pregastrulation stages. Cell $2010 ; 143: 617-27$.

34. Vallier L, Alexander M, Pedersen RA. Activin/Nodal and FGF pathways cooperate to maintain pluripotency of human embryonic stem cells. J Cell Sci $2005 ; 118: 4495-509$.

35. Ludwig TE, Levenstein ME, Jones JM, et al. Derivation of human embryonic stem cells in defined conditions. Nat Biotechnol $2006 ; 24: 185-7$.
36. Daheron L, Opitz SL, Zaehres $\mathrm{H}$, et al. LIF/STAT3 signaling fails to maintain self-renewal of human embryonic stem cells. Stem Cells $2004 ; 22: 770-8$.

37. Takashima Y, Guo G, Loos R, et al. Resetting transcription factor control circuitry toward ground-state pluripotency in human. Cell 2014 ; 158 : 125469.

38. Chen H, Aksoy I, Gonnot F, et al. Reinforcement of STAT3 activity reprogrammes human embryonic stem cells to naïve-like pluripotency. Nat Commun $2015 ; 6: 7095-112$.

39. Sperber $\mathrm{H}$, Mathieu J, Wang $\mathrm{Y}$, et al. The metabolome regulates the epigenetic landscape during naive-to-primed human embryonic stem cell transition. Nat Cell Biol 2015; $17: 1523-35$.

40. Rada-Iglesias A, Bajpai R, Swigut T, et al. A unique chromatin signature uncovers early developmental enhancers in humans. Nature $2010 ; 470$ : 279-83.

41. Nakamura T, Okamoto I, Sasaki K, et al. A developmental coordinate of pluripotency among mice, monkeys and humans. Nature 2016 ; 537 : 57-62

42. Mascetti VL, Pedersen RA. Human-mouse chimerism validates human stem cell pluripotency. Cell Stem Cell 2016; 18:67-72.

43. Masaki H, Kato-Itoh M, Umino A, et al. Interspecific in vitro assay for the chimera-forming ability of human pluripotent stem cells. Development $2015 ; 142: 3222-30$.

44. Guo G, von Meyenn F, Santos F, et al. Naive pluripotent stem cells derived directly from isolated cells of the human inner cell mass. Stem Cell Reports $2016 ; 6: 437-46$.

45. Huang K, Maruyama T, Fan G. The naive state of human pluripotent stem cells: a synthesis of stem cell and preimplantation embryo transcriptome analyses. Cell Stem Cell $2014 ; 15: 410-5$.

46. Manor YS, Massarwa R, Hanna JH. Establishing the human naive pluripotent state. Curr Opin Genet Dev $2015 ; 34: 35-45$

47. Weinberger L, Ayyash M, Novershtern N, Hanna JH. Dynamic stem cell states: naive to primed pluripotency in rodents and humans. Nat Rev Mol Cell Biol $2016 ; 17: 155-69$

48. Warrier $S$, Van der Jeught $M$, Duggal $G$, et al. Direct comparison of distinct naive pluripotent states in human embryonic stem cells. Nat Commun 2017 ; $8: 15055$.

49. Collier AJ, Rugg-Gunn PJ. Identifying human naive pluripotent stem cells Evaluating state-specific reporter lines and cell-surface markers. Bioessays 2018 : el700239.

50. Boroviak T, Nichols J. Primate embryogenesis predicts the hallmarks of human naive pluripotency. Development 2017 ; 144 : 175-86.

51. Petropoulos $S$, Edsgard D, Reinius B, et al. Single-cell RNA-Seq reveals lineage and $X$ chromosome dynamics in human preimplantation embryos. Cell $2016 ; 165: 1012-26$

52. Funk WD, Labat I, Sampathkumar J, et al. Evaluating the genomic and sequence integrity of human $\varepsilon S$ cell lines; comparison to normal genomes. Stem Cell Res $2012 ; 8$ : 154-64.

53. $\mathrm{Na}$ J, Baker D, Zhang J, et al. Aneuploidy in pluripotent stem cells and implications for cancerous transformation. Protein Cell $2014 ; 5: 569-79$.

54. Masaki H, Nakauchi H. Interspecies chimeras for human stem cell research. Development 2017 ; 144 : 2544-7.

55. Wu J, Greely HT, Jaenisch R, et al. Stem cells and interspecies chimaeras. Nature 2016 ; $540: 51-9$

\section{TIRÉS À PART}

P. Savatier

\section{LA FONDATION PREMUP : UN OPÉRATEUR DE TERRAIN EN PÉRINATALITÉ RECONNU POUR SON EXCELLENCE ET SON INTERDISCIPLINARITÉ}

La Fondation de coopération scientifique PremUp, unique en Europe, intervient sur la prévention du handicap à la naissance, par la protection de la santé de la femme enceinte et du nouveau-né.

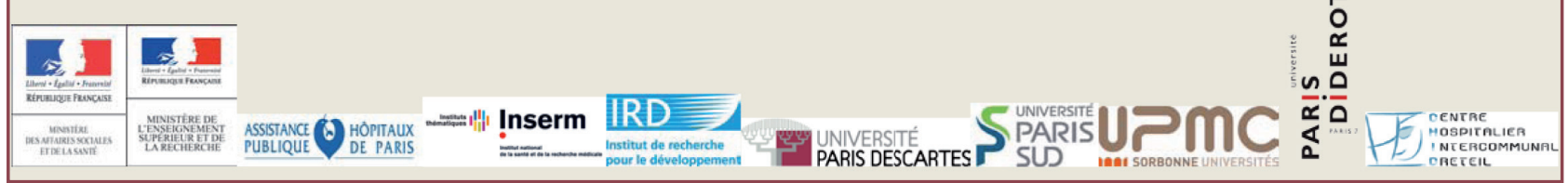

